\title{
A case report of sustained clinical remission in patients with locally advanced lung adenocarcinoma after sequential immunotherapy following concurrent chemoradiotherapy
}

\author{
Weilin Chen, Haoyi Zhang, Weifeng Huang, Tingting Lan \\ Department of Radiation Oncology, Zhangzhou Affiliated Hospital of Fujian Medical University, Zhangzhou, China \\ Correspondence to: Weilin Chen. Department of Radiation Oncology, Zhangzhou Affiliated Hospital of Fujian Medical University, Zhangzhou 363000, \\ China. Email: cwlqiao@163.com.
}

\begin{abstract}
Lung cancer is the most common cause of cancer-related deaths worldwide. Pathologically, lung cancer can be non-small cell lung cancer (NSCLC) or small cell lung cancer (SCLC), while NSCLC accounts for approximately $85 \%$ of lung cancer patients. Stage III NSCLC represents a heterogeneous group of disease entities that are potentially curable and are usually dealt with multimodality treatments involving radiotherapy, chemotherapy, and surgical resection. Immune checkpoint inhibitors (ICIs) target programmed cell death receptor-1 (PD-1) and programmed death-ligand 1 (PD-L1). Studies have shown that ICIs have excellent and long-lasting anti-cancer effects in many cancers. The PACIFIC study is the first in the systemic treatment of stage III unresectable NSCLC in the past few decades that both progression-free survival (PFS) and overall survival (OS) have obtained positive results, However, the performance of this treatment strategy remains to be studied in a real-world setting. Such as who will benefit from treatment is still worthy of our continuous exp loration. In this paper, a patient with locally advanced unresectable NSCLC who underwent concurrent chemoradiotherapy followed by sequential immunotherapy (durvalumab) was reported. The patient obtained sustained clinical benefits despite low PD-L1 expression. This case report may serve as a reference for clinicians to make diagnostic and treatment decisions in clinical practice.
\end{abstract}

Keywords: Lung adenocarcinoma; concurrent chemoradiotherapy; immunotherapy; clinical remission

Submitted Jun 16, 2020. Accepted for publication Oct 24, 2020.

doi: 10.21037/apm-20-1773

View this article at: http://dx.doi.org/10.21037/apm-20-1773

\section{Introduction}

According to the Cancer Statistics, 2019, lung cancer is the second most common malignancy and the leading cause of cancer-related deaths in both men and women (1). In China, lung cancer is the most prevalent and lethal malignancy (2). Non-small cell lung cancer (NSCLC) accounts for approximately $85 \%$ of lung cancer patients, and approximately one-third of NSCLC patients are in stage III at the time of diagnosis (3). For patients with stage III unresectable NSCLC who are in good physical conditions, standard of care is concurrent radiotherapy and platinum-based doublet chemotherapy (chemoradiotherapy) (4). However, most patients have local relapse or distant metastasis during the follow-up period after chemoradiotherapy, and the 5-year survival rate is only $15-30 \%$, which means that the median survival is less than 28 months (4). The poor long-term survival for unresectable stage III NSCLC patients, as a result of the subsequent progression and metastasis of the residual disease following definitive chemoradiation, has been a major challenge that demands an effective consolidation treatment. Several studies have investigated the efficacy of systemic treatments in patients whose condition is under control after chemoradiotherapy. However, so far, the treatments have been proven to be ineffective, and the median survival after consolidation treatment is only 18 to 23 months (5-9). 

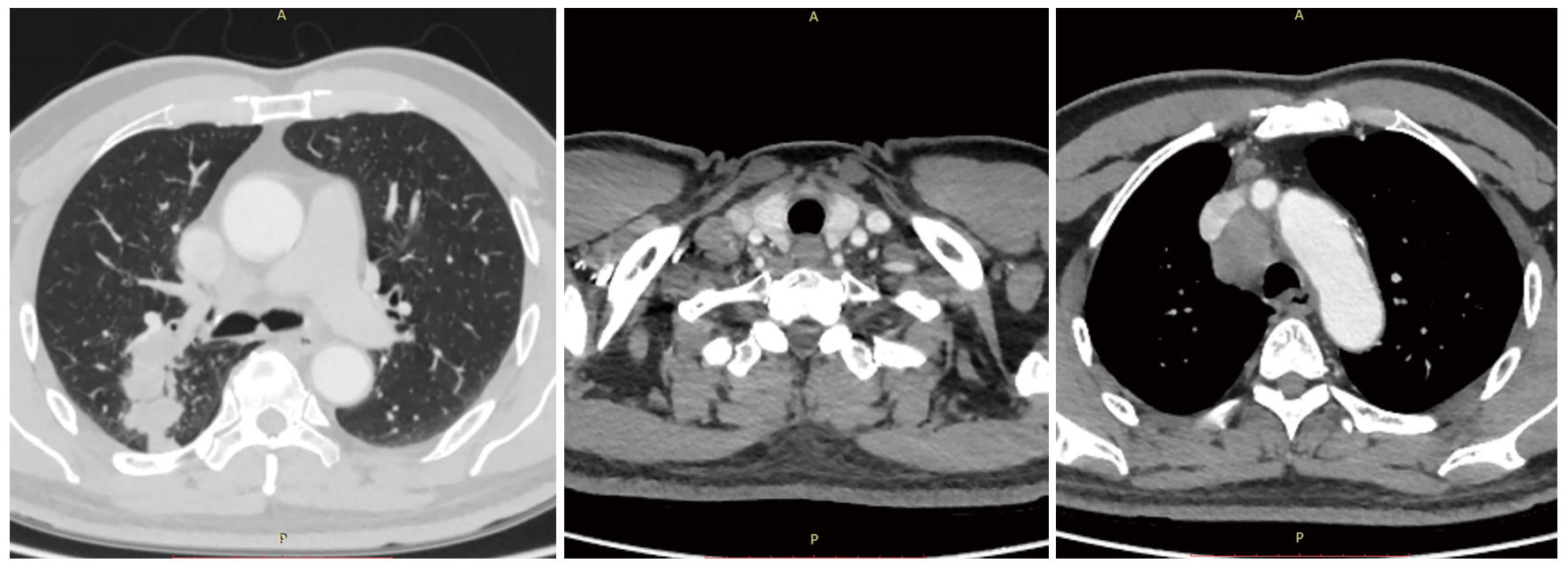

Figure 1 Primary lung lesions $(51 \mathrm{~mm} \times 21 \mathrm{~mm})$ and right supraclavicular and mediastinal metastatic lymph nodes $(37 \mathrm{~mm} \times 28 \mathrm{~mm})$ before concurrent chemoradiotherapy.

Durvalumab, a selective human immunoglobulin G1 (IgG1) monoclonal antibody with high affinity, blocks programmed death receptor-1 (PD-1) from binding to programmed death-ligand 1 (PD-L1) and cluster of differentiation 80 (CD80), thereby allowing $\mathrm{T}$ cells to recognize and kill tumor cells (10-12). PACIFIC is a placebo-controlled, multicenter, randomized, double-blind, phase III clinical trial to evaluate the efficacy of durvalumab, an immune checkpoint inhibitor (ICI), in patients with unresectable phase III NSCLC who have no disease progression after concurrent chemoradiotherapy (13). The first set of study data were published in $N$ Engl 7 Med in September 2017 (13). The results showed that progression-free survival (PFS), one of the two primary endpoints, was significantly longer in the durvalumab group than in the placebo group [median PFS: 16.8, 95\% confidence interval (CI): $13.0-18.1$ vs. $5.6,95 \%$ CI: 4.67.8 , and the odds ratio (OR) of progression or death was 0.52 (95\% CI: $0.42-0.65, \mathrm{P}<0.001$ ). Based on these results, durvalumab was approved for the treatment of unresectable stage III NSCLC without progression after concurrent platinum-based chemoradiotherapy. In this regard, the PDL1 inhibitor durvalumab given in the wake of concurrent chemoradiation represented a major leap toward the consolidation strategies. In the phase III PACIFIC study, durvalumab administered at six weeks post chemoradiation, compared to the placebo, showed a significantly longer PFS, time to distant metastasis and overall survival (OS). Another phase II study with a similar strategy applied the regimen of PD-1 inhibitor pembrolizumab and has also reported a longer time to distant metastasis compared to the historical controls.

In this paper, the clinical data of a patient with unresectable stage IIIC NSCLC who underwent sequential immunotherapy after concurrent chemoradiotherapy were reported.

We present the following case in accordance with the CARE reporting checklist (available at http://dx.doi. org/10.21037/apm-20-1773).

\section{Case presentation}

In September 2019, a 69-year-old man visited our hospital due to repeated coughing with bloody sputum. Plain and enhanced chest computed tomography (CT) showed (I) a space-occupying lesion in the apical segment of the right upper lobe, suggesting a malignant tumor with multiple lymph node metastases in the right supraclavicular fossa, mediastinum, and right hilum (Figure 1); and (II) obstructive inflammation of the right upper lobe. Plain and enhanced brain + upper abdominal magnetic resonance imaging (MRI) and whole-body bone scan did not show any visible metastasis. After confirming that the patient had no relevant contraindications, endobronchial ultrasound-guided transbronchial needle aspiration (EBUSTBNA) under local anesthesia was performed to further confirm the diagnosis. Bronchoscopy showed a polypoid neoplasm at the opening of the apicoposterior segment 

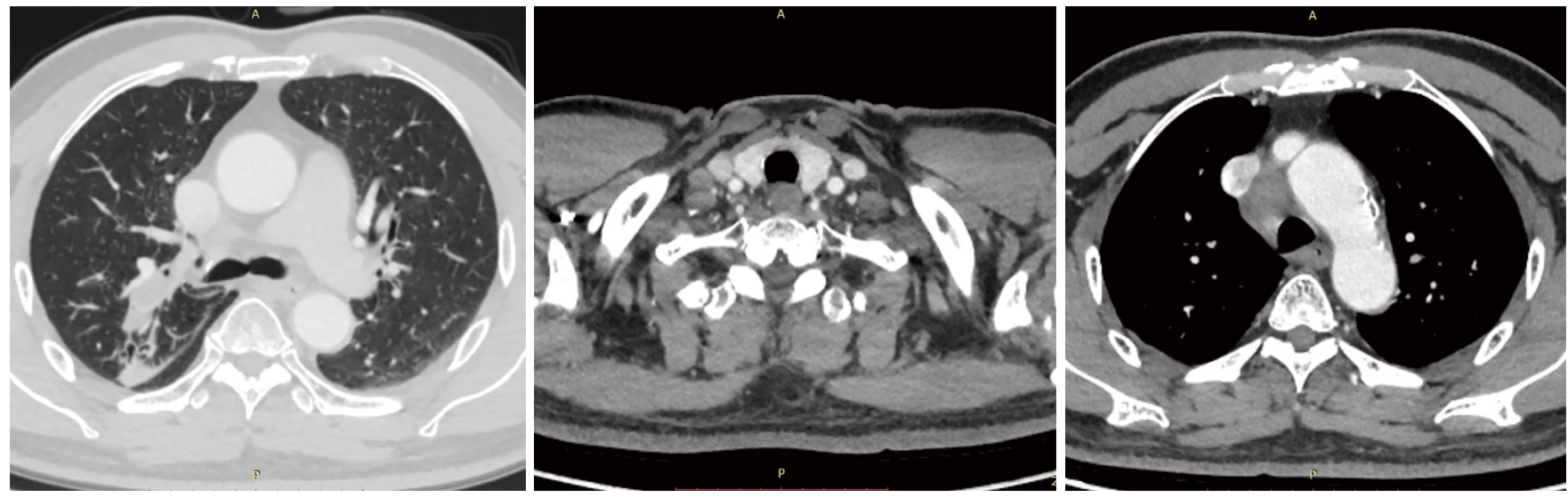

Figure 2 Primary lung lesions $(48 \mathrm{~mm} \times 14 \mathrm{~mm})$ and right supraclavicular and mediastinal metastatic lymph nodes $(28 \mathrm{~mm} \times 20 \mathrm{~mm})$ after concurrent chemoradiotherapy.

of the upper right lobe. The neoplasm blocked the lumen and was prone to bleeding upon touch. Postprocedural pathology showed poorly differentiated cancer, which, combined with immunohistochemistry, confirmed NSCLC (poorly differentiated adenocarcinoma). Additional immunohistochemistry results indicated CD56 (-) and vimentin (-). Genetic testing showed no mutations on EGFR, KRAS, ALK, BRAF, ROS1, MET, RET, ERBB2, NTRK, or PIK3CA. The tumor proportion score (TPS) for PD-L1 expression was approximately $2 \%$. The patient had a long-term history of heavy smoking [heaviness of smoking index (HSI): 1,500]. He had hypertension and diabetes and had been regularly taking blood pressure and diabetes drugs for a long time. Final diagnosis was poorly differentiated adenocarcinoma of the right upper lobe with multiple lymph node metastases in the right supraclavicular fossa, mediastinum, and right hilum (cT3N3M0, stage IIIc). The patient underwent radical concurrent chemoradiotherapy from September 30, 2019 to October 29, 2019, and target area was as follows: gross tumor volume (GTV) was determined based on right lung lesions, as well as right supraclavicular, right hilar, and mediastinal $2 \mathrm{R}, 4 \mathrm{R}$, and 7 metastatic lymph nodes on CT; planning target volume (PTV) was extended $0.8 \mathrm{~cm}$ beyond GTV. Prescribed dose was as follows: for PTV, DT 60 Gy/24 F, along with concurrent chemotherapy for two 3 -week cycles (drug administration was on day 1 of each cycle (September 30 and October 31, 2019) with pemetrexed $500 \mathrm{mg} / \mathrm{m}^{2}$ ivgtt + cisplatin $80 \mathrm{mg} / \mathrm{m}^{2}$ ivgtt. The Pacific study group analysis show us the middle and early stages of central (less than 14 days) Immunotherapy intervention resulted in longer
PFS and OS. Pre-immunotherapy baseline evaluation included autoimmune diseases, infectious diseases, intestinal function, blood tests, skin and mucous membranes, cardiopulmonary function, and endocrine function. No absolute contraindications were identified. On November 11,2019 , the patient started to take durvalumab $740 \mathrm{mg}$ on day 1 of each 2-week cycle, with a total of 12 cycles (last treatment: April 15, 2020).

The patient and his family members were informed about the efficacy and potential adverse reactions of concurrent chemoradiotherapy, and sequential immunotherapy; and the informed consent was signed before treatment. All procedures performed in studies involving human participants were in accordance with the ethical standards of the institutional and/or national research committee(s) and with the Helsinki Declaration (as revised in 2013).

\section{Results}

During concurrent chemoradiotherapy, coughing and bloody sputum were gradually improved. After chemoradiotherapy, chest CT on October 30, 2019 showed that the space-occupying lesion in the apical segment of the right upper lobe shrank to approximately $48 \mathrm{~mm} \times 14 \mathrm{~mm}$ from approximately $51 \mathrm{~mm} \times 21 \mathrm{~mm}$ before treatment. Moreover, obstructive inflammation of the right upper lobe was improved, metastatic lymph nodes in the right supraclavicular fossa, mediastinum, and right hilum were smaller than before, and the large one was reduced to approximately $28 \mathrm{~mm} \times 20 \mathrm{~mm}$ from approximately $37 \mathrm{~mm}$ $\times 28 \mathrm{~mm}$ (Figure 2). 

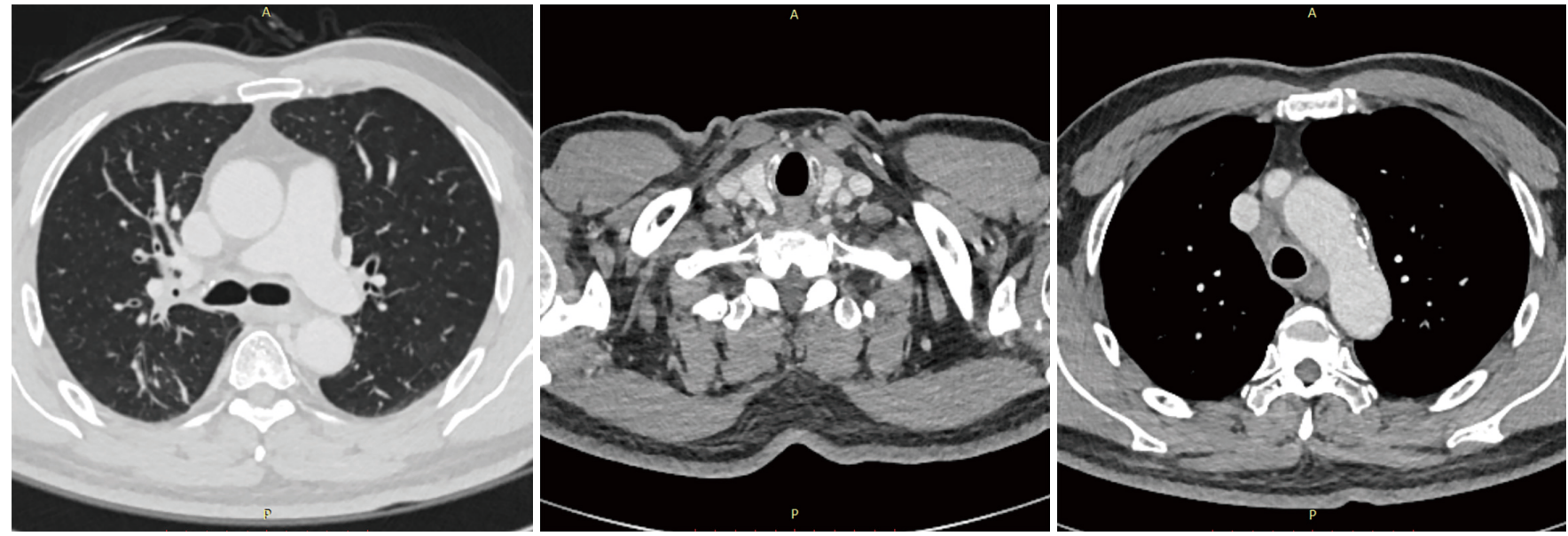

Figure 3 Primary lung lesions $(22 \mathrm{~mm} \times 9.5 \mathrm{~mm})$ and right supraclavicular and mediastinal metastatic lymph nodes $(21 \mathrm{~mm} \times 12.5 \mathrm{~mm})$ after 3 cycles of sequential immunotherapy.
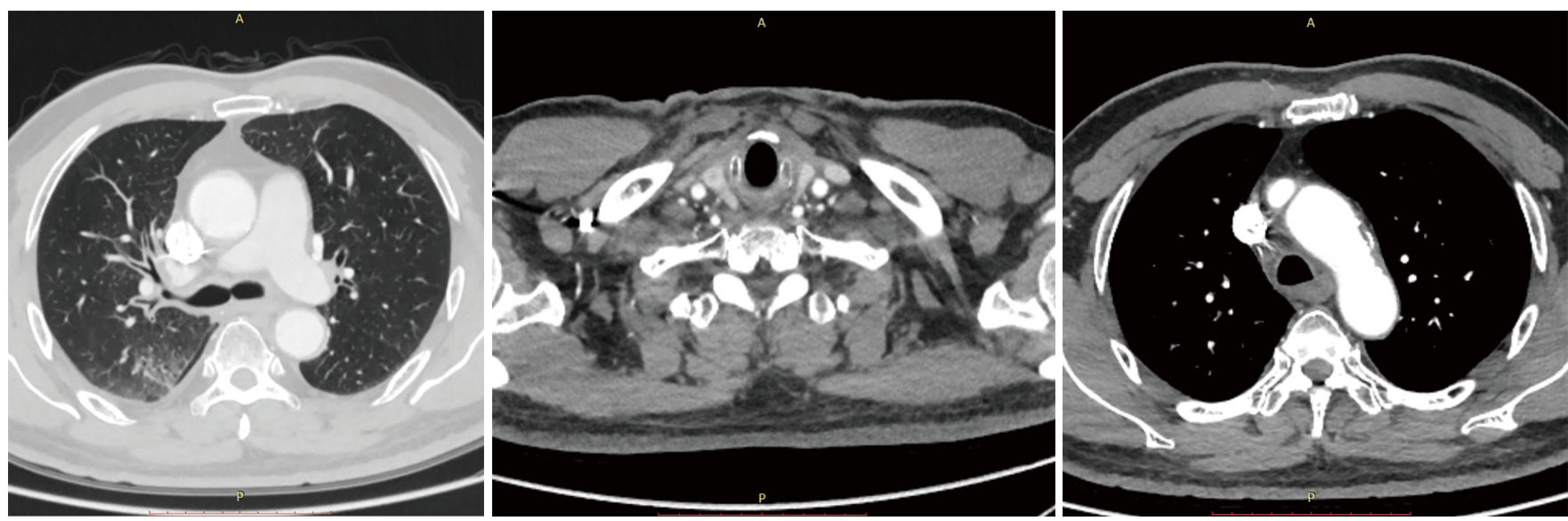

Figure 4 Primary lung lesions $(20.2 \mathrm{~mm} \times 9.3 \mathrm{~mm})$ and right supraclavicular and mediastinal metastatic lymph nodes $(21 \mathrm{~mm} \times 12.5 \mathrm{~mm})$ after 9 cycles of sequential immunotherapy.

After 3 cycles of immunotherapy, chest CT on December 10, 2019 showed that the space-occupying lesion in the apical segment of the right upper lobe shrank to approximately $22 \mathrm{~mm} \times 9.5 \mathrm{~mm}$. Moreover, obstructive inflammation of the right upper lobe continued to improve, and metastatic lymph nodes in the right supraclavicular fossa, mediastinum, and right hilum were smaller than before, with the large one of approximately $21 \mathrm{~mm} \times$ $12.5 \mathrm{~mm}$ (Figure 3).

After 9 cycles of immunotherapy, chest CT on March 4, 2020 showed that the space-occupying lesion in the apical segment of the right upper lobe shrank to approximately $20.2 \mathrm{~mm} \times 9.3 \mathrm{~mm}$. Moreover, obstructive inflammation of the right upper lobe continued to improve, and metastatic lymph nodes in the right supraclavicular fossa, mediastinum, and right hilum were smaller than before, with the large one of approximately $21 \mathrm{~mm} \times 12.5 \mathrm{~mm}$ (Figure 4).

\section{Discussion}

Durvalumab is a human PD-L1 monoclonal antibody that binds to PD-L1 to block the interaction of PD-L1 with PD-1 and CD80 receptors, thereby blocking suppression of PD-L1 on immune responses.

The follow-up data of PACIFIC study were presented at the 2019 annual American Society of Clinical Oncology 
Changes in primary tumor lesions and metastatic lymph nodes

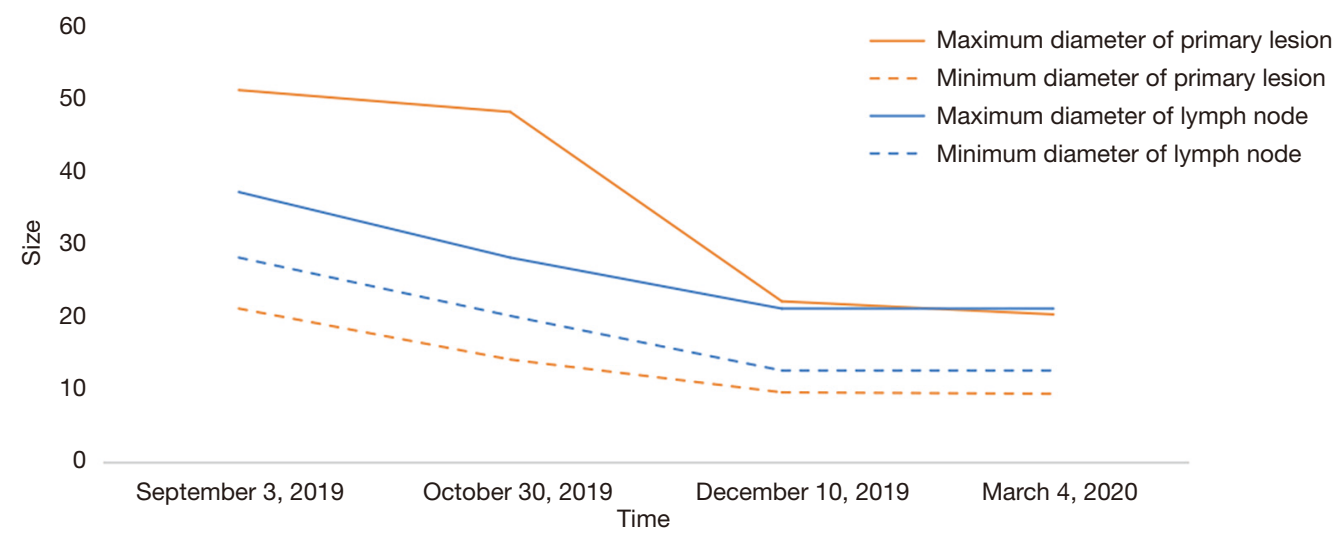

Figure 5 The size of primary tumor lesions and metastatic lymph nodes before and after treatment.

(ASCO) conference, which showed promising benefits of durvalumab consolidation therapy after concurrent chemoradiotherapy (14). Durvalumab improved the 3-year survival rate by $13.5 \%$ (57\% vs. $43.5 \%$ ) and significantly improved overall survival (OS). Moreover, the median time to death or distant metastasis was nearly doubled in the durvalumab group compared to that in the placebo group (28.3 vs. 16.2 months), and the incidence of brain metastases was decreased by nearly $50 \%$ in the durvalumab group (6.3\% vs. 11.8\%). Among patients with stage III NSCLC who received durvalumab consolidation treatment, $1.4 \%$ achieved complete remission (CR) and $27.1 \%$ achieved partial remission (PR), suggesting that durvalumab may be an effective non-surgical treatment in stage III patients. While $27 \%$ of the patients enrolled in PACIFIC study are Asians, there are no data on Chinese populations. The results of the study indicate that durvalumab has clinical benefits, regardless of the PD-L1 expression, although the subgroup analysis shows that high PD-L1 expression is associated with better treatment outcomes. In this case report, ongoing CT scans showed continuous improvement in both primary lesions and local metastatic lymph nodes with sustained PR during sequential immunotherapy after concurrent chemoradiotherapy (Figure 5). Of note, the patient obtained significant clinical benefits despite low PD-L1 expression (2\%). Preclinical studies show that chemotherapy and radiotherapy may up-regulate PD-L1 expression in tumor cells (15-17). Unfortunately, a second biopsy was not performed to examine PD-L1 expression in tumor tissue after chemoradiotherapy because the local tumor was significantly reduced, and there was a possibility of tumor necrosis, which may affect the results of a second biopsy.

The safety summary data of PACIFIC study showed that the incidences of grade 3-4 adverse events were 29.9\% and $26.1 \%$, respectively (14). The overall incidence of allcause pneumonia was $33.9 \%(161 / 475)$ in the durvalumab group and $24.8 \%$ (58/234) in the placebo group. For grade 3-4 pneumonia, the incidences were $3.6 \%(17 / 475)$ and $3 \%(7 / 234)$, respectively. Baseline data were comparable between the two groups. However, the incidence of pneumonia appeared to be high among Asians. In the durvalumab group, patients with non-squamous cell carcinoma were more likely to develop pneumonia. The risk of pneumonia was unrelated to treatment outcomes (CR, PR, stable disease, or progressive disease) after previous concurrent chemoradiotherapy, suggesting that the toxicity of the new treatment was statistically similar to that of concurrent chemoradiotherapy alone. Moreover, no significant intergroup differences were observed in immune-related myocarditis or other immune-related adverse reactions. In this case report, the patient did not show any pneumonia-related clinical symptoms or imaging changes during the follow-up period. The patient was closely monitored for ICI-related life-threatening adverse reactions with laboratory tests, including troponin, myocardial enzymes, brain natriuretic peptide (BNP), and cortisol. No significant adverse reactions or laboratory abnormalities were identified so far. We have seen similar studies reported by other researchers, in which CR, PR, SD and PD were $0(0 / 0), 25.8 \%(8 / 29), 54.8 \%(17 / 29)$ and $12.9 \%(4 / 29)$, respectively, and $13.8 \%$ (4/29) of the patients 
had grade 3-5 severe adverse reactions (18). Therefore, how to screen the effective population and monitor the clinical adverse reaction is still worth the attention of clinicians .

Durvalumab is showing promises in treating lung cancers according to exciting data from POSEIDON, PACIFIC (19), and CASPIAN (20); it may become a safe and effective treatment option. In the future, more effective and comprehensive new treatments may become available to improve the survival, the quality of life, and treatment outcomes of cancer patients. Additionally, clinicians need to continuously improve the ability of early identification and timely treatment of ICIS-related severe organ adverse reactions, so as to maximize the clinical benefits to patients.

\section{Acknowledgments}

Funding: None.

\section{Footnote:}

Reporting Checklist: The authors have completed the CARE reporting checklist. Available at http://dx.doi.org/10.21037/ apm-20-1773

Conflicts of Interest: All authors have completed the ICMJE uniform disclosure form (available at http://dx.doi. org/10.21037/apm-20-1773). The authors have no conflicts of interest to declare.

Ethical Statement: The authors are accountable for all aspects of the work in ensuring that questions related to the accuracy or integrity of any part of the work are appropriately investigated and resolved. The patient and his family members were informed about the efficacy and potential adverse reactions of concurrent chemoradiotherapy, and sequential immunotherapy; and the informed consent was signed before treatment. All procedures performed in studies involving human participants were in accordance with the ethical standards of the institutional and/or national research committee(s) and with the Helsinki Declaration (as revised in 2013).

Open Access Statement: This is an Open Access article distributed in accordance with the Creative Commons Attribution-NonCommercial-NoDerivs 4.0 International License (CC BY-NC-ND 4.0), which permits the noncommercial replication and distribution of the article with the strict proviso that no changes or edits are made and the original work is properly cited (including links to both the formal publication through the relevant DOI and the license). See: https://creativecommons.org/licenses/by-nc-nd/4.0/.

\section{References}

1. Siegel RL, Miller KD, Jemal A. Cancer statistics, 2019. CA Cancer J Clin 2019;69:7-34.

2. Chen $W$, Zheng R, Baade PD, et al. Cancer statistics in China, 2015. CA Cancer J Clin 2016;66:115-32.

3. Aupérin A, Le Pechoux C, Rolland E, et al. Meta-analysis of concomitant versus sequential radiochemotherapy in locally advanced non-small-cell lung cancer. J Clin Oncol 2010;28:2181-90.

4. Yoon SM, Shaikh T, Hallman M. Therapeutic management options for stage III non-small cell lung cancer. World J Clin Oncol 2017;8:1-20.

5. Ahn JS, Ahn YC, Kim JH, et al. Multinational Randomized Phase III Trial With or Without Consolidation Chemotherapy Using Docetaxel and Cisplatin After Concurrent Chemoradiation in Inoperable Stage III NonSmall-Cell Lung Cancer: KCSG-LU05-04. J Clin Oncol 2015;33:2660-6.

6. Skrzypski M, Jassem J. Consolidation systemic treatment after radiochemotherapy for unresectable stage III nonsmall cell lung cancer. Cancer Treat Rev 2018;66:114-21.

7. Tsujino K, Kurata T, Yamamoto S, et al. Is consolidation chemotherapy after concurrent chemo-radiotherapy beneficial for patients with locally advanced non-small-cell lung cancer? A pooled analysis of the literature. J Thorac Oncol 2013;8:1181-9.

8. Kelly K, Chansky K, Gaspar LE, et al. Phase III trial of maintenance gefitinib or placebo after concurrent chemoradiotherapy and docetaxel consolidation in inoperable stage III non-small-cell lung cancer: SWOG S0023. J Clin Oncol 2008;26:2450-6.

9. Hanna N, Neubauer M, Yiannoutsos C, et al. Phase III study of cisplatin, etoposide, and concurrent chest radiation with or without consolidation docetaxel in patients with inoperable stage III non-small-cell lung cancer: the Hoosier Oncology Group and U.S. Oncology. J Clin Oncol 2008;26:5755-60.

10. Stewart R, Morrow M, Hammond SA, et al. Identification and Characterization of MEDI4736, an Antagonistic Anti-PD-L1 Monoclonal Antibody. Cancer Immunol Res 2015;3:1052-62.

11. Pardoll DM. The blockade of immune checkpoints in cancer immunotherapy. Nat Rev Cancer 2012;12:252-64. 
12. Postow MA, Callahan MK, Wolchok JD. Immune Checkpoint Blockade in Cancer Therapy. J Clin Oncol 2015;33:1974-82.

13. Antonia SJ, Villegas A, Daniel D, et al. Durvalumab after Chemoradiotherapy in Stage III Non-Small-Cell Lung Cancer. N Engl J Med 2017;377:1919-29.

14. Gray JE, Villegas A, Daniel D, et al. Three-Year Overall Survival with Durvalumab after Chemoradiotherapy in Stage III NSCLC-Update from PACIFIC. J Thorac Oncol 2020;15:288-93.

15. Zhang P, Su DM, Liang M, et al. Chemopreventive agents induce programmed death-1-ligand 1 (PD-L1) surface expression in breast cancer cells and promote PD-L1mediated T cell apoptosis. Mol Immunol 2008;45:1470-6.

16. Deng L, Liang H, Burnette B, et al. Irradiation and antiPD-L1 treatment synergistically promote antitumor

Cite this article as: Chen W, Zhang $\mathrm{H}$, Huang W, Lan T. A case report of sustained clinical remission in patients with locally advanced lung adenocarcinoma after sequential immunotherapy following concurrent chemoradiotherapy. Ann Palliat Med 2020;9(6):4346-4352. doi: 10.21037/apm-20-1773 immunity in mice. J Clin Invest 2014;124:687-95.

17. Dovedi SJ, Adlard AL, Lipowska-Bhalla G, et al. Acquired resistance to fractionated radiotherapy can be overcome by concurrent PD-L1 blockade. Cancer Res 2014;74:5458-68.

18. Chu CH, Chiu TH, Wang CC, et al. Consolidation treatment of durvalumab after chemoradiation in realworld patients with stage III unresectable non-small cell lung cancer. Thorac Cancer 2020;11:1541-9.

19. Antonia SJ. Durvalumab after Chemoradiotherapy in Stage III Non-Small-Cell Lung Cancer. Reply. N Engl J Med 2019;380:990.

20. Mozaffarian N, Heshmat R, Ataie-Jafari A, et al. Association of sleep duration and snack consumption in children and adolescents: The CASPIAN-V study. Food Sci Nutr 2020;8:1888-97. 